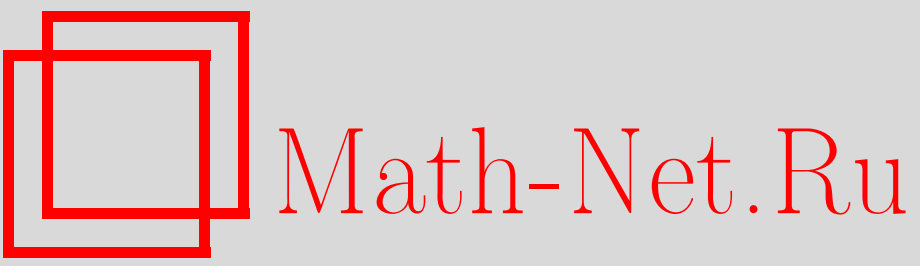

T. Grbić, E. Pap, Generalization of Portmanteau theorem with respect to the pseudoweak convergence of random closed sets, Теория вероятн. и ее примен., 2009, том 54, выпуск 1, 97-115

DOI: https://doi.org/10.4213/tvp2548

Использование Общероссийского математического портала Math-Net.Ru подразумевает, что вы прочитали и согласны с пользовательским соглашением

http://www . mathnet.ru/rus/agreement

Параметры загрузки:

IP: 18.208 .226 .222

26 апреля 2023 г., 03:06:05 
ТЕОРИЯ ВЕРОЯТНОСТЕЙ

Tом 54

И ЕЕ ПРИМЕНЕНИЯ

Выпуск 1

2009

(c) 2009 г. $\quad$ GRBIĆ T.* ${ }^{*}$ PAP E.**

\title{
GENERALIZATION OF PORTMANTEAU THEOREM WITH RESPECT TO THE PSEUDO-WEAK CONVERGENCE OF RANDOM CLOSED SETS ${ }^{1)}$
}

\begin{abstract}
Основным результатом настоящей работы является теорема типа теоремы А.Д. Александрова о слабой сходимости (the portmanteau theorem) для псевдослабо сходящихся последовательностей функционалов емкости для последовательности случайных замкнутых множеств. Для этого классический интеграл Лебега заменяется на более общий, известный как общий псевдоинтеграл, и вводится понятие псевдослабой сходимости функционалов емкости. Устанавливается связь между слабой сходимостью последовательности вероятностных мер, индуцированных последовательностью случайных замкнутых множеств, и сходимостью псевдоинтеграла относительно соответствующей последовательности функционалов емкости.
\end{abstract}

Ключевые слова и фразы: теорема А. Д. Александрова о слабой сходимости (the portmanteau theorem), псевдооперации, псевдоинтеграл, случайное замкнутое множество, функционал емкости, псевдослабая сходимость.

1. Introduction. Theory of random sets has been independently introduced by Kendall [10] and Matheron [17], and they have laid down its foundations. Their starting investigations were related to the stochastic geometry. This theory is based on probability measures on the space of closed subsets of locally compact Hausdorff and separable space endowed with hitor-miss topology. Random closed sets have been introduced as generalizations of random variables, i.e., random closed sets are random elements on the space of closed subsets. Random set theory play important role in image

* Faculty of Technical Sciences, University of Novi Sad, Trg. Dositeja Obradovića 6, 21000 Novi Sad, Serbia; e-mail: tatjana@uns.ns.ac.yu

** Faculty of Science and Mathematics, University of Novi Sad, Trg. Dositeja Obradovića 4, 21000 Novi Sad, Serbia; e-mail: endre.pap@im.ns.ac.yu

1) This work was supported by the Project MNZŽSS144012. The second author was supported also by the grant of MTA HTMT, French-Serbian project «Pavle Savić», and by the project «Mathematical Models for Decision Making under Uncertain Conditions and Their Applications» supported by Vojvodina Provincial Secretariat for Science and Technological Development. 
processing, mathematical morphology, expert system, theoretical statistic, etc., see [8], [9], [17]-[20], [29]. Present theory of random closed sets is closely related to Choquet's capacity theorem [3], and the special important properties of random closed sets are consequences of the topological structure of the space of closed sets. We remark that random sets are actually a special type of correspondence [11], namely, correspondence between probability space and real line endowed with hit-or-miss topology. As it is well known, a sequence of probability measures $\left\{\mathbf{P}_{n}\right\}_{n \in \mathbf{N}}$ converges weakly to a probability measure $\mathbf{P}$ if $\int f d \mathbf{P}_{n} \rightarrow \int f d \mathbf{P}$ for all continuous bounded real functions $f$. Several conditions equivalent to the weak convergence are contained in the portmanteau theorem [2].

Theorem 1.1 (the portmanteau theorem). Let $\left\{\mathbf{P}_{n}\right\}_{n \in \mathbf{N}}$ and $\mathbf{P}$ be probability measures on the same probability space. The following conditions are equivalent:

(i) Sequence of probability measures $\left\{\mathbf{P}_{n}\right\}_{n \in \mathbf{N}}$ converges weakly to probability measure $\mathbf{P}$;

(ii) $\lim _{n \rightarrow \infty} \int f d \mathbf{P}_{n}=\int f d \mathbf{P}$ for all bounded, uniformly continuous real functions $f$;

(iii) $\lim \sup _{n} \mathbf{P}_{n}(F) \leqslant \mathbf{P}(F)$ for all closed sets $F$;

(iv) $\liminf \inf _{n} \mathbf{P}_{n}(G) \geqslant \mathbf{P}(G)$ for all open sets $G$;

(v) $\lim _{n} \mathbf{P}_{n}(A)=\mathbf{P}(A)$ for all $\mathbf{P}$-continuity sets $A$.

While the classical case considers weak convergence of sequences of probability measures, the main result presented here is a theorem of portmanteau type for pseudo-weakly convergent sequences of capacity functionals for sequence of random closed sets, see [6]. To achieve this goal, the classical Lebesgue integral had been substituted with more general one, known as general pseudo integral [1], which is based on pseudo-addition and pseudomultiplication (as generalizations of the classical real operations) and monotone set functions (nonadditive measures), see [25], [26]. One of the basic important cases is the idempotent analysis, see [15], [16].

Section 2 contains preliminary notions, such as pseudo-addition, pseudodifference, pseudo-multiplication, and pseudo-integral (see [1], [12], [15], [16], $[25]$, [26]). Section 3 contains results on separation functionals, which are used in the proofs in Section 5. Some basic definitions and propositions of random closed sets are given in Section 4, where also the important definition of pseudo-weak convergence is introduced. Generalizations of the portmanteau theorem for sequence of random closed sets, i.e., the corresponding sequence of capacity functionals, are given in Section 5 . Connection between weak convergence of sequence of probability measures induced by the sequence of random closed sets and convergence of pseudo-integral with respect to the corresponding sequence of capacity functionals is described in Section 6 . 
2. Pseudo-operations and a pseudo-integral. Real operations used in this paper are based on the approach of [1], [12], [25]. More general case, as well as a wide range of applications have been investigated in [4], [14]-[16], [25], [26]. Let $\leqslant$ be a total order on $[0, \infty]$.

D e f i n i t i o $n$ 2.1. A binary operation $\oplus:[0, \infty]^{2} \rightarrow[0, \infty]$ such that the following conditions are satisfied:

(A1) $a \oplus b=b \oplus a$ (commutativity);

(A2) $a \leqslant a^{\prime} \wedge b \leqslant b^{\prime} \Rightarrow a \oplus b \leqslant a^{\prime} \oplus b^{\prime}$ (monotonicity);

(A3) $(a \oplus b) \oplus c=a \oplus(b \oplus c)$ (associativity);

(A4) $a \oplus 0=a$ (neutral element);

(A5) $a_{n} \rightarrow a \wedge b_{n} \rightarrow b \Rightarrow\left(a_{n} \oplus b_{n}\right) \rightarrow a \oplus b$ (continuity);

is called pseudo-addition.

Example 2.1. The following operations are pseudo-additions (see [1], [12], [16], [25], [26]):

(i) An idempotent pseudo-addition is $a \oplus b=\max (a, b)$;

(ii) For an increasing bijection $g:[0, \infty] \rightarrow[0, \infty]$, pseudo-addition is defined by $a \oplus b=g^{-1}(g(a)+g(b))$.

The following general representation of the pseudo-addition can be found in [12], [25], [26].

Theorem 2.1. A binary operation $\oplus:[0, \infty]^{2} \rightarrow[0, \infty]$ is a pseudoaddition from Definition 2.1 if and only if there exist a family $\left\{\left(\alpha_{k}, \beta_{k}\right)\right\}_{k \in K}$ of pairwise disjoint intervals contained in $[0, \infty]$, and a family $\left\{g_{k}\right\}_{k \in K}$ of functions $g_{k}:\left[\alpha_{k}, \beta_{k}\right] \rightarrow[0, \infty]$ which are continuous strictly increasing and $g_{k}\left(\alpha_{k}\right)=0$ for all $k \in K$ such that

$$
a \oplus b= \begin{cases}g_{k}^{*}\left(g_{k}(a)+g_{k}(b)\right), & (a, b) \in\left[\alpha_{k}, \beta_{k}\right]^{2}, \\ \max \{a, b\}, & \text { otherwise }\end{cases}
$$

where $g_{k}^{*}$ is the pseudo-inverse of $g_{k}$ given by $g_{k}^{*}(x)=g_{k}^{-1}\left(\min \left\{x, g_{k}\left(\beta_{k}\right)\right\}\right)$.

$\mathrm{R}$ e $\mathrm{m}$ a r k 2.1. Theorem 2.1 implies the following consequences.

(i) If $g_{k}\left(\beta_{k}\right)=\infty$, then $g_{k}^{*}=g_{k}^{-1}$. Functions $g_{k}$ are determined uniquely by $\oplus$ up to a positive multiplicative constant.

(ii) Each pseudo-addition from Definition 2.1 is an ordinal sum (which may have infinitely many summands) given by generators (each of summands can have different generator), i.e., $\oplus=\left\{\left\langle\left(\alpha_{k}, \beta_{k}\right), g_{k}\right\rangle\right\}_{k \in K}$ (see [5], [12], [25]).

For the completeness of the paper we give a statement with a proof related to real monotone functions.

Proposition 2.1. If $H:[0, \infty]^{2} \rightarrow[0, \infty]$ is a continuous nondecreasing function and $\left\{x_{n}\right\}_{n \in \mathbf{N}}$ and $\left\{y_{n}\right\}_{n \in \mathbf{N}}$ are sequences from $[0, \infty]$, then

(i) $\lim \sup _{n \rightarrow \infty} H\left(x_{n}, y_{n}\right) \leqslant H\left(\lim \sup _{n \rightarrow \infty} x_{n}, \lim \sup _{n \rightarrow \infty} y_{n}\right)$,

(ii) $\liminf _{n \rightarrow \infty} H\left(x_{n}, y_{n}\right) \geqslant H\left(\liminf _{n \rightarrow \infty} x_{n}, \liminf _{n \rightarrow \infty} y_{n}\right)$. 
P r o o f. (i) The continuity and monotonicity of the function $H$ imply

$$
\begin{gathered}
\limsup _{n \rightarrow \infty} H\left(x_{n}, y_{n}\right)=\limsup _{k \rightarrow \infty} H\left(x_{n}, y_{n}\right) \leqslant \lim _{k \rightarrow \infty} \sup _{n \geqslant k} \sup _{m \geqslant k} H\left(x_{n}, y_{m}\right) \\
=\lim _{k \rightarrow \infty} H\left(\sup _{n \geqslant k} x_{n}, \sup _{m \geqslant k} y_{m}\right)=H\left(\lim _{k \rightarrow \infty} \sup _{n \geqslant k} x_{n}, \lim _{k \rightarrow \infty} \sup _{m \geqslant k} y_{m}\right) \\
=H\left(\limsup _{n \rightarrow \infty} x_{n}, \limsup _{m \rightarrow \infty} y_{m}\right)=H\left(\limsup _{n \rightarrow \infty} x_{n}, \limsup _{n \rightarrow \infty} y_{n}\right) .
\end{gathered}
$$

(ii) The proof is analogous to the proof of (i).

Corollary 2.1. Let $\oplus$ be a pseudo-addition and let $\left\{x_{n}\right\}_{n \in \mathbf{N}}$ and $\left\{y_{n}\right\}_{n \in \mathbf{N}}$ be sequences from $[0, \infty]$, then

$$
\begin{aligned}
\limsup _{n} x_{n} \oplus \limsup _{n} y_{n} & \geqslant \limsup _{n}\left(x_{n} \oplus y_{n}\right), \\
\liminf _{n} x_{n} \oplus \liminf _{n} y_{n} & \leqslant \liminf _{n}\left(x_{n} \oplus y_{n}\right) .
\end{aligned}
$$

For $a>b$ the equation $b \oplus x=a$ always has a solution, however this solution need not be unique. The following definition of a pseudo-difference, see [1], [12], is used through this paper.

$\mathrm{D}$ efinition 2.2. The pseudo-difference is the binary operation $\ominus:[0, \infty]^{2} \rightarrow[0, \infty]$ given by $a \ominus b=\inf \{x \in[0, \infty]: b \oplus x \geqslant a\}$.

$\mathrm{Ex}$ a $\mathrm{m} \mathrm{p} \mathrm{l} \mathrm{e} \mathrm{2.2.} \mathrm{For} \mathrm{pseudo-additions} \mathrm{from} \mathrm{Example} 2.1$ the corresponding pseudo-differences are given by

$$
\text { (i) } a \ominus b=\left\{\begin{array}{ll}
0, & a \leqslant b, \\
a, & a>b,
\end{array} \quad \text { (ii) } a \ominus b= \begin{cases}0, & a \leqslant b, \\
g^{-1}(g(a)-g(b)), & a>b,\end{cases}\right.
$$

respectively.

The following lemma describes some basic properties of the pseudodifference (see [1], [12]).

Lemma 2.1. For all $a, b, c \in[0, \infty]$ we have:

(i) $a \ominus b=0$ if $a \leqslant b$,

(ii) $a \ominus b>0$ if $a>b$,

(iii) if $a \geqslant b$, then

$$
b \oplus(a \ominus b)=a,
$$

(iv) $a \ominus b \leqslant a$,

(v) $a<b \Rightarrow a \ominus c \leqslant b \ominus c$,

(vi) $b<c \Rightarrow a \ominus b \geqslant a \ominus c$.

$\mathrm{D}$ e $\mathrm{f}$ i $\mathrm{n}$ i t i o $\mathrm{n}$ 2.3. For a given pseudo-addition $\oplus$ the corresponding pseudo-multiplication is a binary operation $\odot:[0, \infty]^{2} \rightarrow[0, \infty]$ such that the following conditions are satisfied:

(M1) $a \odot b=b \odot a$ (commutativity),

(M2) $a \odot 0=0$ (zero element), 
(M3) $a \leqslant a^{\prime} \wedge b \leqslant b^{\prime} \Rightarrow a \odot b \leqslant a^{\prime} \odot b^{\prime}$ (monotonicity),

(M4) $(a \oplus b) \odot c=(a \odot c) \oplus(a \odot c)$ (distributivity),

(M5) $a \odot \mathbf{1}=a$ (unit element),

(M6) $a \odot(b \odot c)=(a \odot b) \odot c$ (associativity),

(M7) $a_{n} \rightarrow a \wedge b_{n} \rightarrow b \Rightarrow\left(a_{n} \odot b_{n}\right) \rightarrow a \odot b$ (continuity).

$\mathrm{Ex}$ a m p l e 2.3. (i) For pseudo-addition from Example 2.1 (i) one of possible pseudo-multiplications is $a \odot b=a \cdot b$, see [14], [16].

(ii) For the pseudo-addition from Example 2.1 (ii) the corresponding pseudo-multiplications is $a \odot b=g^{-1}(g(a) g(b))$, see [12], [25], [26].

$\mathrm{R}$ e $\mathrm{m}$ a r k 2.2. (i) The algebraic structure $([0, \infty], \oplus, \odot)$ is a semiring, see [5], [13].

(ii) Pseudo-operations can be defined on the closed subinterval $[a, b] \subseteq$ $[-\infty,+\infty]$ (or, in some cases, semiclosed interval) as in [25], [26]. In this case, $\preceq$ is a total order on $[a, b]$ and the pseudo-addition is a function $\oplus:[a, b]^{2} \rightarrow$ $[a, b]$ which is commutative, nondecreasing (with respect to $\preceq$ ), associative and with zero element denoted by $\mathbf{0}$. Pseudo-multiplication is a function $\odot:[a, b]^{2} \rightarrow[a, b]$ which is commutative, positively nondecreasing $(x \preceq y$ implies $x \odot z \preceq y \odot z$ for $\left.z \in[a, b]_{+}=\{x \in[a, b]: \mathbf{0} \preceq z\}\right)$, associative and with unit element, denoted by $\mathbf{1}$. Additionally, $\mathbf{0} \odot x=\mathbf{0}$ and $x \odot(y \oplus z)=$ $(x \odot y) \oplus(x \odot z)$.

Let $\mathscr{A}$ be a $\sigma$-algebra of subsets of nonempty abstract space $\Omega$ and $m: \mathscr{A} \rightarrow \mathbf{R}$ nondecreasing set function with $m(\varnothing)=0$. Let $\mathscr{M}$ be a family of $\mathscr{A}$-measurable functions $f: \Omega \rightarrow[0, \infty]$. A measurable function $s: \Omega \rightarrow[0, \infty]$ is called a simple function if its range is finite. Let Rang $(s)=\left\{a_{1}, \ldots, a_{k}\right\}$, where $0<a_{1}<\cdots<a_{k}$, and $A_{i} \cap A_{j}=\varnothing$ for $i \neq j$. The standard $\oplus$-step representation of a simple function $s$ is

$$
s=\bigoplus_{i=1}^{k} b\left(c_{i}^{*}, C_{i}^{*}\right)
$$

where $c_{1}^{*}=a_{1}, c_{2}^{*}=a_{2} \ominus a_{1}, \ldots, c_{k}^{*}=a_{k} \ominus a_{k-1}, C_{i}^{*}=\bigcup_{j=i}^{k} A_{j}$, and $b: \Omega \rightarrow$ $[0, \infty]$ is a basic function given by

$$
b\left(c_{i}^{*}, C_{i}^{*}\right)(\omega)= \begin{cases}c_{i}^{*}, & \omega \in C_{i}^{*}, \\ 0, & \omega \notin C_{i}^{*} .\end{cases}
$$

Specially, for $c_{i}^{*}=\mathbf{1}$ and $A \in \mathscr{A}$, the basic function reduces to the pseudo characteristic function $\chi_{A}: \Omega \rightarrow\{0, \mathbf{1}\}$ given by

$$
\chi_{A}(\omega)= \begin{cases}1, & \omega \in A \\ 0, & \omega \notin A .\end{cases}
$$


D e f i n it i o n 2.4. (a) The pseudo-integral of a simple function $s$ with the standard $\oplus$-step representation (2.4) is given by

$$
\int^{\oplus} s \odot d m=\bigoplus_{i=1}^{k} c_{i}^{*} \odot m\left(C_{i}^{*}\right) .
$$

(b) The pseudo-integral of a function $f \in \mathscr{M}$ is

$$
\int^{\oplus} f \odot d m=\sup \left\{\int^{\oplus} s \odot d m: s \in \mathscr{S}_{f}\right\},
$$

where $\mathscr{S}_{f}$ is a family of all simple functions $s$ such that $s \leqslant f$.

Some of the basic properties of the pseudo-integral are:

Ge.1. ${ }^{\oplus} b(c, C) \odot d m=c \odot m(C)$ (basic values),

Ge.2. $f \leqslant g \Rightarrow \int^{\oplus} f \odot d m \leqslant \int^{\oplus} g \odot d m$ (monotonicity),

Ge.3. $\int{ }^{\oplus} \chi_{A} \odot d m=m(A)$ (reconstruction of the measure).

$\mathrm{Ex}$ a m ple 2.4. (i) For $\oplus=\max$ and $\odot=$. the corresponding integral of simple function $s$ with the standard $\oplus$-step representation (2.4) is given by

$$
\int^{\oplus} s \odot d m=\max _{i=1}^{m}\left(c_{i}^{*} \cdot m\left(C_{i}^{*}\right)\right) .
$$

If $m$ is $\sigma$ - $\oplus$-measure, then there exists a function $h: \mathbf{R} \rightarrow[0, \infty]$ such that $m(A)=\sup _{x \in A} h(x)$ (see [25]). In this case, pseudo-integral of bounded, measurable function $f$ has the following form:

$$
\int^{\oplus} f \odot d m=\sup _{x \in \Omega}(f(x) \cdot h(x)) .
$$

(ii) For pseudo-operations given by generator $g$, the corresponding integral of simple function $s$ with the standard $\oplus$-step representation (2.4) is given by

$$
\int^{\oplus} s \odot d m=g^{-1}\left(\sum_{i=1}^{k} g\left(c_{i}^{*}\right) \cdot g\left(m\left(C_{i}^{*}\right)\right)\right) .
$$

If $m$ is a $\sigma$ - $\oplus$-measure, then pseudo-integral of bounded, measurable function $f$ is given by

$$
\int_{[c, d]}^{\oplus} f \odot d m=g^{-1}\left(\int_{[c, d]} g \circ f d(g \circ m)\right)
$$

where $[c, d] \subset[0, \infty]$, see $[25]$. 
3. Separation functionals. Let $\oplus, \ominus$, and $\odot$ be pseudo-operations from Definitions 2.1, 2.2, and 2.3, respectively. Let $\mathscr{I}$ denote the interval $[0, \infty]$ endowed with operations $\oplus, \ominus$, and $\odot$. Let $1^{*}$ be an element from $[0, \infty]$ such that $1^{*} \odot 1=\mathbf{1}$. Let $F$ and $G$ be Borel subsets of $\mathbf{R}$ and $d$ distance from $x$ to $F$ given by

$$
d(x, F)=\inf \{\rho(x-y): y \in F\},
$$

where $\rho$ is a metric on $\mathbf{R}$.

Theorem 3.1. If $F$ is a closed subset of $\mathbf{R}$ and $\varepsilon>0$, then there exists a function $f_{\varepsilon}: \mathbf{R} \rightarrow \mathscr{I}$ such that

1) $f_{\varepsilon}(x)=\mathbf{1}$ for $x \in F$,

2) $f_{\varepsilon}(x)=0$ if $d(x, F)>\varepsilon$,

3) $0 \leqslant f_{\varepsilon}(x) \leqslant 1$ for all $x$.

P r o o f. Consider a function $\varphi: \mathbf{R} \rightarrow \mathscr{I}$ of the following form:

$$
\varphi(t)= \begin{cases}\mathbf{1}, & t \leqslant 0, \\ \mathbf{1} \ominus t, & 0<t<1, \\ 0, & t \geqslant 1 .\end{cases}
$$

First, we have to show that $\varphi$ maps $\mathbf{R}$ into $[0, \mathbf{1}]$. Obviously, for $t \leqslant 0$ and $t \geqslant 1$ this claim holds. Now, let us consider the case $t \in(0,1)$.

(i) While $t \geqslant \mathbf{1}$, from the definition of pseudo-difference it follows immediately that $\mathbf{1} \ominus t=0$.

(ii) For $t \in(0, \mathbf{1})$, Lemma 2.1 give us $\mathbf{1} \ominus t \leqslant \mathbf{1}$. Also, for $t<\mathbf{1}$ we have $\mathbf{1} \ominus t>0$ (this follows from the definition of pseudo-difference and the monotonicity of pseudo-addition).

Therefore, $\varphi$ maps $\mathbf{R}$ in $[0, \mathbf{1}]$.

The next step is to show that $\varphi$ is nonincreasing function, i.e., $\varphi\left(t_{1}\right) \geqslant$ $\varphi\left(t_{2}\right)$ while $t_{1}<t_{2}$ and $t_{1}, t_{2} \in(0,1)$ (generally, $t_{1}, t_{2} \in \mathbf{R}$, however $t_{1}, t_{2} \in$ $(0,1)$ is the only nontrivial case). Since the cases $\mathbf{1}<t_{1}<t_{2}$ and $t_{1}<\mathbf{1}<t_{2}$ are trivial $\left(\mathbf{1} \ominus t_{1}=0=\mathbf{1} \ominus t_{2}\right.$ for the first one, and $\mathbf{1} \ominus t_{1}=0$ and $\mathbf{1} \ominus t_{2}>0$ for the second one), let us consider the case $t_{1}<t_{2}<\mathbf{1}$. Since $t_{1}<t_{2}$, Lemma 2.1 give us $\mathbf{1} \ominus t_{1} \geqslant \mathbf{1} \ominus t_{2}$ i.e., $\varphi\left(t_{1}\right) \geqslant \varphi\left(t_{2}\right)$.

Let $d$ be the distance on $\mathbf{R}$ given by (3.1), $F$ closed subset of $\mathbf{R}$, and $\varepsilon>0$. The function $f_{\varepsilon}: \mathbf{R} \rightarrow \mathscr{I}$ given by $f_{\varepsilon}(x)=\varphi\left(\frac{1}{\varepsilon} d(x, F)\right)$ has all required properties:

1) for $x \in F$ it holds $d(x, F)=0$ and $f_{\varepsilon}(x)=\varphi\left(\frac{1}{\varepsilon} \cdot 0\right)=\varphi(0)=\mathbf{1}$;

2) if $d(x, F)>\varepsilon$, then $\varphi\left(\frac{1}{\varepsilon} d(x, F)\right) \leqslant \varphi\left(\frac{1}{\varepsilon} \varepsilon\right)$ (because $\varphi$ is nonincreasing function) and therefore

$$
0 \leqslant f_{\varepsilon}(x)=\varphi\left(\frac{1}{\varepsilon} d(x, F)\right) \leqslant \varphi\left(\frac{1}{\varepsilon} \varepsilon\right)=\varphi(1)=0 ;
$$

3) from the construction of function $\varphi$ it follows that $f_{\varepsilon}: \mathbf{R} \rightarrow[0, \mathbf{1}]$.

We have the analogous theorem for open sets. 
Theorem 3.2. If $G$ is an open subset of $\mathbf{R}$ and $\varepsilon>0$, then there exist a function $g_{\varepsilon}: \mathbf{R} \rightarrow \mathscr{I}$ such that

1) $g_{\varepsilon}(x)=\mathbf{1}$ if $d\left(x, G^{c}\right)>\varepsilon$,

2) $g_{\varepsilon}(x)=0$ for $x \in G^{c}$,

3) $0 \leqslant g_{\varepsilon}(x) \leqslant \mathbf{1}$ for all $x$.

P r o o f. Let $1^{*}$ be an element from $[0, \infty]$ such that $1^{*} \odot 1=\mathbf{1}$.

Let us consider function $\psi: \mathbf{R} \rightarrow \mathscr{I}$ of the form

$$
\psi(t)= \begin{cases}0, & t \leqslant 0, \\ 1^{*} \odot t, & 0<t<1, \\ \mathbf{1}, & t \geqslant 1 .\end{cases}
$$

First, let us show that function $\psi$ maps $\mathbf{R}$ into the interval $[0, \mathbf{1}]$. This holds for $t \leqslant 0$ or $t \geqslant 1$, so let $t \in(0,1)$. Suppose that the opposite claim holds, i.e., $\psi(t) \notin(0, \mathbf{1})$.

(i) The first option is $\psi(t) \geqslant \mathbf{1}$. The definition of the function $\psi$ yields $1^{*} \odot t \geqslant \mathbf{1}$, and monotonicity and associativity of the pseudo-multiplication and $1^{*} \odot 1=1$ imply

$1 \odot\left(1^{*} \odot t\right) \geqslant 1 \odot \mathbf{1} \Leftrightarrow\left(1 \odot 1^{*}\right) \odot t \geqslant 1 \odot \mathbf{1} \Leftrightarrow \mathbf{1} \odot t \geqslant \mathbf{1} \odot 1 \Leftrightarrow t \geqslant 1$, which contradicts the initial assumption $t \in(0,1)$.

(ii) The second option is $\psi(t) \leqslant 0$. The definition of the function $\psi$ gives us $1^{*} \odot t \leqslant 0$. Since the pseudo-multiplication is a monotone operation, we have $1 \odot\left(1^{*} \odot t\right) \leqslant 1 \odot 0$. Associativity of the pseudo-multiplication, $1 \odot 1^{*}=\mathbf{1}, 1 \odot 0=0$, and $\mathbf{1} \odot t=t$ give us $t \leqslant 0$, which is, again, a contradictory to the initial assumption $t \in(0,1)$.

Thus, $\psi(t) \in(0, \mathbf{1})$.

The next step is to show that $\psi$ is a monotone nonincreasing function, i.e., $\psi\left(t_{1}\right) \geqslant \psi\left(t_{2}\right)$ while $t_{1}<t_{2}$ and $t_{1}, t_{2} \in(0,1)$ (generally, $t_{1}, t_{2} \in \mathbf{R}$, however $t_{1}, t_{2} \in(0,1)$ is the only nontrivial case). Since pseudo-multiplication is a monotone operation, $1^{*} \odot t_{1} \leqslant 1^{*} \odot t_{2}$ follows from $t_{1}<t_{2}$, and, due to the definition of the function $\psi$, we have $\psi\left(t_{1}\right) \leqslant \psi\left(t_{2}\right)$.

Let $G$ be an open subset of $\mathbf{R}$, let $d$ be the distance on $\mathbf{R}$ given by (3.1) and $\varepsilon>0$. Function $g_{\varepsilon}$ defined by $g_{\varepsilon}(x)=\psi\left(\varepsilon^{-1} d\left(x, G^{c}\right)\right)$ has all of the required properties:

1) if $d\left(x, G^{c}\right)>\varepsilon$, then, due to the monotonicity of $\psi$,

$$
\mathbf{1} \geqslant g_{\varepsilon}(x)=\psi\left(\frac{1}{\varepsilon} d\left(x, G^{c}\right)\right) \geqslant \psi\left(\frac{1}{\varepsilon} \cdot \varepsilon\right)=\psi(1)=\mathbf{1} ;
$$

2) for $x \in G^{c}$ we have $d\left(x, G^{c}\right)=0$ and

$$
g_{\varepsilon}(x)=\psi\left(\varepsilon^{-1} d\left(x, G^{c}\right)\right)=\psi\left(\varepsilon^{-1} \cdot 0\right)=\psi(0)=0 ;
$$

3 ) from the construction of the function $\psi$ it follows that $0 \leqslant g_{\varepsilon}(x) \leqslant \mathbf{1}$ for all $x$. 
4. Pseudo-weak convergence of random closed sets and capacity functionals. We recall some basic notions from the theory of random sets [7], [17], [21]-[24]. Collections of closed, open, and compact subsets of $\mathbf{R}$ are denoted by $\mathscr{F}, \mathscr{O}$, and $\mathscr{K}$, respectively. Of the special importance for the theory of random sets is the collection $\mathscr{F}$ of closed sets, as well as its sub-collections $\mathscr{F}_{G}, G \in \mathscr{O}$, and $\mathscr{F}^{K}, K \in \mathscr{K}$, defined in the following way:

$$
\begin{aligned}
\mathscr{F}_{G} & =\{F \in \mathscr{F}: F \cap G \neq \varnothing\}, & G \in \mathscr{O}, \\
\mathscr{F}^{K} & =\{F \in \mathscr{F}: F \cap K=\varnothing\}, & K \in \mathscr{K} .
\end{aligned}
$$

The collections $\left\{\mathscr{F}_{G}: G \in \mathscr{O}\right\}$ and $\left\{\mathscr{F}^{K}: K \in \mathscr{K}\right\}$ generate a topology $\tau(\mathscr{F})$ on $\mathscr{F}$ (hit-or-miss-topology). $\mathscr{F}$ with the hit-or-miss topology is a compact, separable Hausdorff space [17]. Taking countable unions and intersections of the open sets of the topological space $(\mathscr{F}, \tau(\mathscr{F}))$, a $\sigma$-field $\Sigma(\mathscr{F})$ is generated in $\mathscr{F}$. By [7], [17], [21], [22] we have the following definition.

$\mathrm{D}$ e f i n it i o n 4.1. A random closed set $\mathbf{S}$ is a measurable mapping from the probability space $(\Omega, \mathscr{A}, \mathbf{P})$ into the measurable space $(\mathscr{F}, \Sigma(\mathscr{F}))$.

A random closed set $\mathbf{S}$ generates probability distribution $\mathbf{P}_{\mathbf{S}}$ in the following way:

$$
\mathbf{P}_{\mathbf{S}}(A)=\mathbf{P}(\{\omega \in \Omega: \mathbf{S}(\omega) \in A\})=\mathbf{P}_{\mathbf{S}}(\mathbf{S} \in A), \quad \text { for all } \quad A \in \Sigma(\mathscr{F}) .
$$

The probability distribution $\mathbf{P}_{\mathbf{S}}$ of the random closed set $\mathbf{S}$ is determined by functional $\mathbf{T}_{\mathbf{S}}$ defined on the space of compact subsets of $\mathbf{R}$.

$\mathrm{D}$ e f i $\mathrm{n}$ i t i o $\mathrm{n}$ 4.2. The capacity functional $\mathbf{T}_{\mathbf{S}}: \mathscr{K} \rightarrow[0,1]$ of a random closed set $\mathbf{S}$ for $K \in \mathscr{K}$ is defined by

$$
\mathbf{T}_{\mathbf{S}}(K)=\mathbf{P}_{\mathbf{S}}\left(\mathbf{S} \in \mathscr{F}_{K}\right)=\mathbf{P}_{\mathbf{S}}(\mathbf{S} \cap K \neq \varnothing)
$$

Although the capacity functional $\mathbf{T}_{\mathbf{S}}$ given by (4.1) is defined only on $\mathscr{K}$, it can be extended onto the family $\mathscr{P}$ of all subsets of $\mathbf{R}$, in the following way:

$$
\mathbf{T}_{\mathbf{S}}^{*}(G)=\sup \left\{\mathbf{T}_{\mathbf{S}}(K): K \in \mathscr{K}, K \subset G\right\}, \quad G \in \mathscr{G},
$$

and

$$
\mathbf{T}_{\mathbf{S}}^{*}(M)=\inf \left\{\mathbf{T}_{\mathbf{S}}^{*}(G): G \in \mathscr{G}, M \subset G\right\}, \quad M \in \mathscr{P} .
$$

A subset $M \subset \mathbf{R}$ is called capacitable if

$$
\mathbf{T}_{\mathbf{S}}(M)=\sup \left\{\mathbf{T}_{\mathbf{S}}(K): K \in \mathscr{K}, K \subset M\right\} .
$$

Obviously, $\mathbf{T}_{\mathbf{S}}^{*}$ coincides with $\mathbf{T}_{\mathbf{S}}$ on $\mathscr{K}$, and all Borel sets $B$ are capacitable [17], [22]. We have by [22] the following theorem.

Theorem 4.1. (i) $\mathbf{T}_{\mathbf{S}}^{*}(K)=\mathbf{T}_{\mathbf{S}}(K)$ for all $K \in \mathscr{K}$.

(ii) For each Borel set $B$ it holds

$$
\mathbf{T}_{\mathbf{S}}^{*}(B)=\sup \left\{\mathbf{T}_{\mathbf{S}}(K): K \in \mathscr{K}, K \subset B\right\} .
$$


Further on, $\mathbf{T}_{\mathbf{S}}^{*}(M)$ for all capacitable sets $M$ will be denoted by $\mathbf{T}_{\mathbf{S}}(M)$.

For a given random closed set $\mathbf{S}$ the corresponding capacity functional is denoted by $\mathbf{T}$. Capacity functionals of the random closed sets are uniquely characterized by the following proposition (see [7], [17], [22], [23]).

Proposition 4.1. (i) For all $K \in \mathscr{K}, 0 \leqslant \mathbf{T}(K) \leqslant 1$ and $\mathbf{T}(\varnothing)=0$.

(ii) For all random closed sets and for all $K_{1}, K_{2} \in \mathscr{K}$,

$$
K_{1} \subseteq K_{2} \Rightarrow \mathbf{T}\left(K_{1}\right) \leqslant \mathbf{T}\left(K_{2}\right) .
$$

(iii) The capacity functional is upper semi-continuous, i.e.,

$$
K_{n} \downarrow K \Rightarrow \mathbf{T}\left(K_{n}\right) \downarrow \mathbf{T}(K), \quad \text { for } \quad K_{n} \in \mathscr{K}, \quad n=1,2, \ldots, \quad K \in \mathscr{K} .
$$

We introduce the following generalization [6].

D e f in ition 4.3. A sequence of capacity functionals $\left\{\mathbf{T}_{n}\right\}_{n \in \mathbf{N}}$ is said to converge $(\oplus, \odot)$-weakly (shortly, to converge pseudo-weakly) to capacity functional $\mathbf{T}$ if and only if

$$
\lim _{n \rightarrow \infty} \int^{\oplus} f \odot d \mathbf{T}_{n}=\int^{\oplus} f \odot d \mathbf{T} .
$$

for each continuous, bounded function $f: \mathbf{R} \rightarrow[0, \infty]$.

$\mathrm{E} x \mathrm{a} \mathrm{m} \mathrm{p} \mathrm{l} \mathrm{e} \mathrm{4.1.} \mathrm{(i)} \mathrm{For} \mathrm{a} \mathrm{random} \mathrm{closed} \mathrm{set} \mathbf{S}=\{\mathbf{X}\}$ and a sequence of random closed sets $\left\{\mathbf{S}_{n}\right\}_{n \in \mathbf{N}}$, where $\mathbf{S}_{n}=\left\{\mathbf{X}_{n}\right\}, \mathbf{X}$ is a random variable, and $\left\{\mathbf{X}_{n}\right\}_{n \in \mathbf{N}}$ is a sequence of random variables, we have $\mathbf{T}(K)=\mathbf{P}(\mathbf{X} \in K)$ and $\mathbf{T}_{n}(K)=\mathbf{P}\left(\mathbf{X}_{n} \in K\right)$ [7], where $\mathbf{T}$ and $\mathbf{T}_{n}$ are capacity functionals of random sets $\mathbf{S}$ and $\mathbf{S}_{n}$, respectively. Since $\mathbf{T}(B)=\sup \{\mathbf{T}(K): K \in$ $\mathscr{K}, K \subset B\}$ for every Borel set $B$ (see [22]), we have $\mathbf{T}(B)=\mathbf{P}(\mathbf{X} \in B)$. For all $n \in \mathbf{N}$ we have $\mathbf{T}_{n}(B)=\mathbf{P}\left(\mathbf{X}_{n} \in B\right)$. In this case equality (4.2) is

$$
\lim _{n \rightarrow \infty} \sup _{s \in \mathscr{S}_{f}} \bigoplus_{m=1}^{k} a_{m} \odot \mathbf{P}\left(\mathbf{X}_{n} \in A_{m}\right)=\sup _{s \in \mathscr{S}_{f}} \bigoplus_{m=1}^{k} a_{m} \odot \mathbf{P}\left(\mathbf{X} \in A_{m}\right),
$$

where $\left\{a_{1}, \ldots, a_{k}\right\}$ is the range of simple function $s$ and $A_{i} \cap A_{j}=\varnothing$ for $i \neq j$.

For $\oplus=\max$ and $\odot=$. the corresponding integral is given in Example 2.4 (i) and equality (4.2) has the following form:

$$
\lim _{n \rightarrow \infty} \sup _{s \in \mathscr{S}_{f}} \max _{m=1}^{k} a_{m} \cdot \mathbf{P}\left(\mathbf{X}_{n} \in A_{m}\right)=\sup _{s \in \mathscr{S}_{f}} \max _{m=1}^{k} a_{m} \cdot \mathbf{P}\left(\mathbf{X} \in A_{m}\right) .
$$

For pseudo-operations given by generator $g$, the corresponding integral is given in Example 2.4 (ii) and equality (4.2) has the following form:

$$
\begin{aligned}
\lim _{n \rightarrow \infty} \sup _{s \in \mathscr{S}_{f}} g^{-1}\left(\sum_{m=1}^{k} g\left(a_{m}\right) \cdot g\left(\mathbf{P}\left(\mathbf{X}_{n} \in A_{m}\right)\right)\right) \\
=\sup _{s \in \mathscr{S}_{f}} g^{-1}\left(\sum_{m=1}^{k} g\left(a_{m}\right) \cdot g\left(\mathbf{P}\left(\mathbf{X} \in A_{m}\right)\right)\right) .
\end{aligned}
$$


(ii) For a random closed set $\mathbf{S}=(-\infty, \mathbf{X}]$ and a sequence of random closed sets $\left\{\mathbf{S}_{n}\right\}_{n \in \mathbf{N}}$, where $\mathbf{S}_{n}=\left(-\infty, \mathbf{X}_{n}\right], \mathbf{X}$ is a random variable and $\left\{\mathbf{X}_{n}\right\}_{n \in \mathbf{N}}$ is a sequence of random variables, we have $\mathbf{T}(K)=\mathbf{P}(\mathbf{X}>\inf K)$ and $\mathbf{T}_{n}(K)=\mathbf{P}\left(\mathbf{X}_{n}>\inf K\right)$ [7], where $\mathbf{T}$ and $\mathbf{T}_{n}$ are capacity functionals of random sets $\mathbf{S}$ and $\mathbf{S}_{n}$, respectively. Since $\mathbf{T}(B)=\sup \{\mathbf{T}(K): K \in$ $\mathscr{K}, K \subset B\}$ for all Borel sets $B$, we have $\mathbf{T}(B)=\mathbf{P}(\mathbf{X}>\inf B)$. Also, for all $n \in \mathbf{N}$ we have $\mathbf{T}_{n}(B)=\mathbf{P}\left(\mathbf{X}_{n}>\inf B\right)$. In this case equality (4.2) is

$$
\lim _{n \rightarrow \infty} \sup _{s \in \mathscr{S}_{f}} \bigoplus_{m=1}^{k} a_{m} \odot \mathbf{P}\left(\mathbf{X}_{n}>\inf A_{m}\right)=\sup _{s \in \mathscr{S}_{f}} \bigoplus_{m=1}^{k} a_{m} \odot \mathbf{P}\left(\mathbf{X}>\inf A_{m}\right),
$$

where $\left\{a_{1}, \ldots, a_{k}\right\}$ is the range of simple function $s$ and $A_{i} \cap A_{j}=\varnothing$ for $i \neq j$.

5. Generalization of the portmanteau theorem. The following three theorems generalize the classical portmanteau theorem (Theorem 1.1).

Theorem 5.1. If a sequence of capacity functionals $\left\{\mathbf{T}_{n}\right\}_{n \in \mathbf{N}}$ pseudoweakly converges to the capacity functional $\mathbf{T}$, then

$$
\limsup _{n} \mathbf{T}_{n}(F) \leqslant \mathbf{T}(F)
$$

for all closed sets $F \subseteq \mathbf{R}$.

P r o of. For $\varepsilon>0$ and a closed set $F$ we consider the function $f_{\varepsilon}: \mathbf{R} \rightarrow[0, \infty]$ from Theorem 3.1 given by $f_{\varepsilon}(x)=\varphi\left(\frac{1}{\varepsilon} d(x, F)\right)$, where $d$ is the distance on $\mathbf{R}$ given by (3.1). For $\varepsilon>0$ we introduce open sets $G_{\varepsilon}=\{x: d(x, F)>\varepsilon\}$ such that $G_{\varepsilon} \downarrow F$ and $\mathbf{T}\left(G_{\varepsilon}\right) \downarrow \mathbf{T}(F)$ as $\varepsilon \rightarrow 0$, i.e., for every $\delta>0$ there exists $\varepsilon>0$ such that $\mathbf{T}\left(G_{\varepsilon}\right)<\mathbf{T}(F)+\delta$, where $\mathbf{T}(F)=\inf \{\mathbf{T}(G): G$ is open and $F \subset G\}$.

The inequalities $\chi_{F} \leqslant f_{\varepsilon} \leqslant \chi_{G_{\varepsilon}}$ and monotonicity of the pseudo-integral yield

$$
\int^{\oplus} f_{\varepsilon} \odot d \mathbf{T} \leqslant \int^{\oplus} \chi_{G_{\varepsilon}} \odot d \mathbf{T}=\mathbf{T}\left(G_{\varepsilon}\right)<\mathbf{T}(F)+\delta,
$$

i.e.,

$$
\int^{\oplus} f_{\varepsilon} \odot d \mathbf{T}<\mathbf{T}(F)+\delta
$$

and

$$
\mathbf{T}_{n}(F)=\int^{\oplus} \chi_{F} \odot d \mathbf{T}_{n} \leqslant \int^{\oplus} f_{\varepsilon} \odot d \mathbf{T}_{n} .
$$

By the pseudo-weak convergence, (5.2), and (5.3) it follows that

$$
\begin{aligned}
\limsup _{n} \mathbf{T}_{n}(F) & \leqslant \limsup _{n} \int^{\oplus} f_{\varepsilon} \odot d \mathbf{T}_{n}=\lim _{n} \int^{\oplus} f_{\varepsilon} \odot d \mathbf{T}_{n} \\
& =\int^{\oplus} f_{\varepsilon} \odot d \mathbf{T}<\mathbf{T}(F)+\delta .
\end{aligned}
$$


Since the strict inequality $\lim \sup _{n} \mathbf{T}_{n}(F)<\mathbf{T}(F)+\delta$ holds for every $\delta>0$, we obtain (5.1).

Theorem 5.2. If a sequence of the capacity functionals $\left\{\mathbf{T}_{n}\right\}_{n \in \mathbf{N}}$ pseudo-weakly converges to the capacity functional $\mathbf{T}$, then

$$
\liminf _{n} \mathbf{T}_{n}(G) \geqslant \mathbf{T}(G)
$$

for all open sets $G$.

$\mathrm{P}$ r o o f. Let $\chi_{G}$ be the characteristic function of the open set $G$. For an arbitrary $\varepsilon>0$ we consider the function $g_{\varepsilon}: \mathbf{R} \rightarrow[0, \infty]$ from Theorem 3.2, given by $g_{\varepsilon}(x)=\varphi\left(\frac{1}{\varepsilon} d\left(x, G^{c}\right)\right)$, where $d$ is the distance on $\mathbf{R}$ given by (3.1). Let $F_{\varepsilon}=\left\{x: d\left(x, G^{c}\right) \geqslant \varepsilon\right\}$ be closed sets such that $F_{\varepsilon} \uparrow G$ and $\mathbf{T}\left(F_{\varepsilon}\right) \uparrow$ $\mathbf{T}(G)$ as $\varepsilon \rightarrow 0$, i.e., for every $\delta>0$ there exists $\varepsilon>0$ such that $\mathbf{T}(G)<$ $\mathbf{T}\left(F_{\varepsilon}\right)+\delta$, where $\mathbf{T}(G)=\sup \{\mathbf{T}(F): F$ is closed and $F \subset G\}$.

Using the inequality $\chi_{F_{\varepsilon}} \leqslant g_{\varepsilon} \leqslant \chi_{G}$ we obtain

$$
\int^{\oplus} g_{\varepsilon} \odot d \mathbf{T} \geqslant \int^{\oplus} \chi_{F_{\varepsilon}} \odot d \mathbf{T}=\mathbf{T}\left(F_{\varepsilon}\right)>\mathbf{T}(G)-\delta,
$$

i.e.,

$$
\int^{\oplus} g_{\varepsilon} \odot d \mathbf{T}>\mathbf{T}(G)-\delta
$$

and

$$
\mathbf{T}_{n}(G)=\int^{\oplus} \chi_{G} \odot d \mathbf{T}_{n} \geqslant \int^{\oplus} g_{\varepsilon} \odot d \mathbf{T}_{n} .
$$

By the pseudo-weak convergence, (5.5), and (5.6) we obtain that

$$
\begin{aligned}
\liminf _{n} \mathbf{T}_{n}(G) & \geqslant \liminf _{n} \int^{\oplus} g_{\varepsilon} \odot d \mathbf{T}_{n}=\lim _{n} \int^{\oplus} g_{\varepsilon} \odot d \mathbf{T}_{n} \\
& =\int^{\oplus} g_{\varepsilon} \odot d \mathbf{T}>\mathbf{T}(G)-\delta .
\end{aligned}
$$

Since for every $\delta>0$ the strict inequality $\lim \inf _{n} \mathbf{T}_{n}(G)>\mathbf{T}(G)-\delta$ holds, we have (5.4).

Theorem 5.3. If $\lim \sup _{n} \mathbf{T}_{n}(F) \leqslant \mathbf{T}(F)$ for all closed sets $F$ and $\liminf _{n} \mathbf{T}_{n}(G) \geqslant \mathbf{T}(G)$ for all open sets $G$, then the sequence of capacity functionals $\left\{\mathbf{T}_{n}\right\}_{n \in \mathbf{N}}$ pseudo-weakly converges to the capacity functional $\mathbf{T}$.

P r o o f. The first two steps in order to prove the claim are to show that inequalities

$$
\begin{aligned}
& \limsup _{n \rightarrow \infty} \int^{\oplus} s \odot d \mathbf{T}_{n} \leqslant \int^{\oplus} s \odot d \mathbf{T}, \\
& \liminf _{n \rightarrow \infty} \int^{\oplus} s \odot d \mathbf{T}_{n} \geqslant \int^{\oplus} s \odot d \mathbf{T}
\end{aligned}
$$


hold for all simple functions.

Let $s$ be a simple function with the range $\operatorname{Rang}(s)=\left\{a_{1}, \ldots, a_{m}\right\}$ such that $a_{1}<\cdots<a_{m}$ and $A_{i} \cap A_{j}=\varnothing$ for $i \neq j$. For each $a_{i}$, the basic function is

$$
b\left(a_{i}, A_{i}\right)(x)= \begin{cases}a_{i}, & x \in A_{i}, \\ 0, & x \notin A_{i},\end{cases}
$$

and the corresponding pseudo-integral is $\int^{\oplus} b\left(a_{i}, A_{i}\right) \odot d \mathbf{T}=a_{i} \odot \mathbf{T}\left(A_{i}\right)$ (see [1]).

Let $c_{1}^{*}=a_{1}, c_{2}^{*}=a_{2} \ominus a_{1}, \ldots, c_{m}^{*}=a_{m} \ominus a_{m-1}$ and $C_{i}^{*}=\bigcup_{j=i}^{m} A_{j}$. Obviously, $C_{1}^{*} \supseteq \cdots \supseteq C_{m}^{*}$. Suppose that $C_{i}^{*}$ are closed sets (if $C_{i}^{*}$ are not closed, sets $F_{i}^{*}=\operatorname{cl} C_{i}^{*}$ can be considered instead). Since $\int^{\oplus} b\left(c_{i}^{*}, C_{i}^{*}\right) \odot$ $d \mathbf{T}_{n}=c_{i}^{*} \odot \mathbf{T}_{n}\left(C_{i}^{*}\right)$ for all $i \in\{1, \ldots, m\}$ and $\lim \sup _{n} \mathbf{T}_{n}(F) \leqslant \mathbf{T}(F)$, we obtain

$$
\begin{aligned}
\underset{n}{\limsup \int^{\oplus} b\left(c_{i}^{*}, C_{i}^{*}\right) \odot d \mathbf{T}_{n}} & =\underset{n}{\limsup } c_{i}^{*} \odot \mathbf{T}_{n}\left(C_{i}^{*}\right) \\
& =c_{i}^{*} \odot \limsup _{n} \mathbf{T}_{n}\left(C_{i}^{*}\right) \leqslant c_{i}^{*} \odot \mathbf{T}\left(C_{i}^{*}\right),
\end{aligned}
$$

i.e.,

$$
\begin{aligned}
& \limsup _{n} c_{1}^{*} \odot \mathbf{T}_{n}\left(C_{1}^{*}\right) \leqslant c_{1}^{*} \odot \mathbf{T}\left(C_{1}^{*}\right), \\
& \limsup c_{2}^{*} \odot \mathbf{T}_{n}\left(C_{2}^{*}\right) \leqslant c_{2}^{*} \odot \mathbf{T}\left(C_{2}^{*}\right), \\
& \text {....................... } \\
& \limsup c_{m}^{*} \odot \mathbf{T}_{n}\left(C_{m}^{*}\right) \leqslant c_{m}^{*} \odot \mathbf{T}\left(C_{m}^{*}\right) .
\end{aligned}
$$

Based on the monotonicity of the pseudo-addition, pseudo-adding previous inequalities we obtain

$$
\begin{aligned}
& \underset{n}{\limsup } c_{1}^{*} \odot \mathbf{T}_{n}\left(C_{1}^{*}\right) \oplus \cdots \oplus \underset{n}{\limsup } c_{m}^{*} \odot \mathbf{T}_{n}\left(C_{m}^{*}\right) \\
& \quad \leqslant c_{1}^{*} \odot \mathbf{T}\left(C_{1}^{*}\right) \oplus c_{2}^{*} \odot \mathbf{T}\left(C_{2}^{*}\right) \oplus \cdots \oplus c_{m}^{*} \odot \mathbf{T}\left(C_{m}^{*}\right) .
\end{aligned}
$$

The right-hand side of the last inequality is a pseudo-integral of the simple function $s$ and, therefore, we have

$$
\limsup _{n} c_{1}^{*} \odot \mathbf{T}_{n}\left(C_{1}^{*}\right) \oplus \cdots \oplus \limsup _{n} c_{m}^{*} \odot \mathbf{T}_{n}\left(C_{m}^{*}\right) \leqslant \int^{\oplus} s \odot d \mathbf{T} .
$$

On the other hand, by the definition of the pseudo-integral and inequality (2.1) we have

$$
\begin{gathered}
\underset{n}{\limsup } \int_{n}^{\oplus} s \odot d \mathbf{T}_{n}=\underset{n}{\limsup }\left(c_{1}^{*} \odot \mathbf{T}_{n}\left(C_{1}^{*}\right) \oplus \cdots \oplus c_{m}^{*} \odot \mathbf{T}_{n}\left(C_{m}^{*}\right)\right) \\
\leqslant \limsup c_{1}^{*} \odot \mathbf{T}_{n}\left(C_{1}^{*}\right) \oplus \cdots \oplus \limsup c_{m}^{*} \odot \mathbf{T}_{n}\left(C_{m}^{*}\right),
\end{gathered}
$$


and (5.7) follows directly from (5.9), (5.10), and the definition of the pseudointegral.

The next step is to prove (5.8). Suppose that sets $C_{i}^{*}$ are open (if $C_{i}^{*}$ are not open, sets $G_{i}^{*}$ such that $C_{i}^{*} \subset G_{i}^{*}$ can be taken instead). Since $\int{ }^{\oplus} b\left(a_{i}, A_{i}\right) \odot d \mathbf{T}=a_{i} \odot \mathbf{T}\left(A_{i}\right)$ and $\liminf _{n} \mathbf{T}_{n}(G) \geqslant \mathbf{T}(G)$, for all $i \in$ $\{1, \ldots, m\}$ we obtain

$$
\begin{aligned}
\liminf _{n} \int^{\oplus} b\left(c_{i}^{*}, C_{i}^{*}\right) \odot d \mathbf{T}_{n} & =\liminf _{n} c_{i}^{*} \odot \mathbf{T}_{n}\left(C_{i}^{*}\right) \\
& =c_{i}^{*} \odot \liminf _{n} \mathbf{T}_{n}\left(C_{i}^{*}\right) \geqslant c_{i}^{*} \odot \mathbf{T}\left(C_{i}^{*}\right) .
\end{aligned}
$$

Pseudo-adding the $m$ inequalities of the form $\liminf _{n} \int{ }^{\oplus} b\left(c_{i}^{*}, C_{i}^{*}\right) \odot d \mathbf{T}_{n} \geqslant$ $c_{i}^{*} \odot \mathbf{T}\left(C_{i}^{*}\right), i \in\{1, \ldots, m\}$, we obtain

$$
\begin{gathered}
\liminf _{n} \int^{\oplus} b\left(c_{1}^{*}, C_{1}^{*}\right) \odot d \mathbf{T}_{n} \oplus \cdots \oplus \liminf _{n} \int^{\oplus} b\left(c_{m}^{*}, C_{m}^{*}\right) \odot d \mathbf{T}_{n} \\
\geqslant c_{1}^{*} \odot \mathbf{T}\left(C_{1}^{*}\right) \oplus \cdots \oplus c_{m}^{*} \odot \mathbf{T}\left(C_{m}^{*}\right) .
\end{gathered}
$$

The right-hand side of the last inequality is the pseudo-integral of the simple function $s$, i.e.,

$\liminf _{n} \int^{\oplus} b\left(c_{1}^{*}, C_{1}^{*}\right) \odot d \mathbf{T}_{n} \oplus \cdots \oplus \liminf _{n} \int^{\oplus} b\left(c_{m}^{*}, C_{m}^{*}\right) \odot d \mathbf{T}_{n} \geqslant \int^{\oplus} s \odot d \mathbf{T}$.

By the definition of the pseudo-integral and inequality (2.2) it follows that

$$
\begin{aligned}
& \liminf _{n} \int^{\oplus} s \odot d \mathbf{T}_{n}=\liminf _{n}\left(c_{1}^{*} \odot \mathbf{T}_{n}\left(C_{1}^{*}\right) \oplus \cdots \oplus c_{m}^{*} \odot \mathbf{T}_{n}\left(C_{m}^{*}\right)\right) \\
& \quad=\liminf _{n}\left(\int^{\oplus} b\left(c_{1}^{*}, C_{1}^{*}\right) \odot d \mathbf{T}_{n} \oplus \cdots \oplus \int^{\oplus} b\left(c_{m}^{*}, C_{m}^{*}\right) \odot d \mathbf{T}_{n}\right) \\
& \quad \geqslant \liminf _{n} \int^{\oplus} b\left(c_{1}^{*}, C_{1}^{*}\right) \odot d \mathbf{T}_{n} \oplus \cdots \oplus \liminf _{n}^{\oplus} b\left(c_{m}^{*}, C_{m}^{*}\right) \odot d \mathbf{T}_{n} .
\end{aligned}
$$

Now, (5.8) is the direct consequence of (5.11), (5.12), and the definition of the pseudo-integral.

Since for every simple function inequalities (5.7) and (5.8) hold, we have $\lim _{n \rightarrow \infty} \int^{\oplus} s \odot d \mathbf{T}_{n}=\int^{\oplus} s \odot d \mathbf{T}$. Since for arbitrary continuous bounded function $f$,

$$
\int^{\oplus} f \odot d \mathbf{T}=\sup \left\{\int^{\oplus} s \odot d \mathbf{T}: s \in \mathscr{S}_{f}\right\},
$$

where $\mathscr{S}_{f}$ is the family of all simple functions $s$ such that $s \leqslant f$, the claim of Theorem 5.3 is valid for all continuous bounded functions $f$.

Corollary 5.1. Let $\mathbf{X}$ be a random variable and let $\left\{\mathbf{X}_{n}\right\}_{n \in \mathbf{N}}$ be a sequence of random variables. For random the closed set $\mathbf{S}$ and the sequence of random closed sets $\left\{\mathbf{S}_{n}\right\}_{n \in \mathbf{N}}$ defined by $\mathbf{S}=\{\mathbf{X}\}$ and $\mathbf{S}_{n}=\left\{\mathbf{X}_{n}\right\}$, pseudoweak convergence is equivalent to the weak convergence with respect to the continuous bounded function $f: \mathbf{R} \rightarrow[0, \infty]$. 
$\mathrm{P}$ r o o f. In this case for any Borel set $B$ and $n \in \mathbf{N}$ we have $\mathbf{T}(B)=$ $\mathbf{P}(\mathbf{X} \in B)$ and $\mathbf{T}_{n}(B)=\mathbf{P}\left(\mathbf{X}_{n} \in B\right)$, see [7], [22]. Let $\left\{\mathbf{S}_{n}\right\}_{n \in \mathbf{N}}$ pseudoweakly converge to $\mathbf{S}$. By Theorem 5.1 it follows that $\lim \sup _{n} \mathbf{P}\left(\mathbf{X}_{n} \in F\right) \leqslant$ $\mathbf{P}(\mathbf{X} \in F)$ for all closed sets $F$. From the portmanteau theorem it follows that $\int f d \mathbf{P}_{n} \rightarrow \int f d \mathbf{P}$, i.e., the sequence of random closed sets $\left\{\mathbf{S}_{n}\right\}_{n \in \mathbf{N}}$ weakly converges to $\mathbf{S}$.

From the weak convergence of the sequence of probability measures, for any open set $G$ we have $\liminf _{n} \mathbf{P}\left(\mathbf{X}_{n} \in G\right) \geqslant \mathbf{P}(\mathbf{X} \in G)$ and for any closed set $F$ we have $\liminf _{n} \mathbf{P}\left(\mathbf{X}_{n} \in F\right) \leqslant \mathbf{P}(\mathbf{X} \in F)$. Then, by Theorem 5.3 the sequence of random closed sets $\left\{\mathbf{S}_{n}\right\}_{n \in \mathbf{N}}$ pseudo-weakly converges to $\mathbf{S}$.

Corollary 5.2. Let random closed set $\mathbf{S}$ and the sequence of random closed sets $\left\{\mathbf{S}_{n}\right\}_{n \in \mathbf{N}}$ be defined in the following way: $\mathbf{S}=(-\infty, \mathbf{X}]$ and $\mathbf{S}_{n}=\left(-\infty, \mathbf{X}_{n}\right]$, where $\mathbf{X}$ is a random variable and $\left\{\mathbf{X}_{n}\right\}_{n \in \mathbf{N}}$ is a sequence of random variables. Then the pseudo-weak convergence implies that for all closed sets $F$ and all open sets $G$

$$
\mathbf{F}(\inf F) \leqslant \liminf _{n} \mathbf{F}_{n}(\inf F) \quad \text { and } \quad \mathbf{F}(\inf G) \geqslant \limsup _{n} \mathbf{F}_{n}(\inf G),
$$

where $\mathbf{F}$ and $\mathbf{F}_{n}$ are corresponding distribution functions of random variables $\mathbf{X}$ and $\mathbf{X}_{n}$, respectively.

P r o o f. From the $(\oplus, \odot)$-weak convergence and Theorem 5.1 it follows that

$$
\begin{aligned}
& \underset{n}{\limsup } \mathbf{T}_{n}(F) \leqslant \mathbf{T}(F), \quad \limsup _{n} \mathbf{P}\left(X_{n}>\inf F\right) \leqslant \mathbf{P}(X>\inf F), \\
& \limsup _{n}\left(1-\mathbf{P}\left(X_{n} \leqslant \inf F\right)\right) \leqslant 1-\mathbf{P}(X \leqslant \inf F), \\
& 1-\liminf _{n} \mathbf{P}\left(X_{n} \leqslant \inf F\right) \leqslant 1-\mathbf{P}(X \leqslant \inf F), \\
& \mathbf{P}(X \leqslant \inf F) \leqslant \liminf _{n} \mathbf{P}\left(X_{n} \leqslant \inf F\right), \quad \mathbf{F}(\inf F) \leqslant \liminf _{n} \mathbf{F}_{n}(\inf F) .
\end{aligned}
$$

Similarly, Theorem 5.2 will give us $\mathbf{F}(\inf G) \geqslant \lim \sup _{n} \mathbf{F}_{n}(\inf G)$.

$\mathrm{R}$ e $\mathrm{m}$ a $\mathrm{rk}$ 5.1. (i) The weak convergence of the sequence of random closed sets with respect to the Choquet integral (the Choquet convergence) is investigated in [23]. The problem studied in [23] is the convergence in distribution of a sequence of random closed sets $\left\{\mathbf{S}_{n}\right\}_{n \in \mathbf{N}}$ to the random closed set $\mathbf{S}$, i.e., the weak convergence of the sequence of probability measures induced by the sequence of random closed sets $\mathbf{S}_{n}$ to the probability measure induced by the random closed set $\mathbf{S}$. Relations between the Choquet convergence and the weak convergence of sequence of random sets were investigated in [23].

(ii) Some equivalent conditions for the weak convergence of the sequence of probability measures induced by the sequence of random closed sets can be found in [24], [27]. 
(iii) The large deviation convergence of a net of probability measures $\left\{\mathbf{P}_{\phi}\right\}_{\phi \in \Phi}$ on $(E, \mathscr{B}(E))$ ( $E$ is a topological space and $\mathscr{B}(E)$ is the Borel $\sigma$-algebra) to the $\mathscr{F}$-idempotent probability $\Pi$ on $E$ is investigated in [28].

Let $\mathscr{S}_{\mathbf{T}}=\{K \in \mathscr{K}: \mathbf{T}(K)=\mathbf{T}(\stackrel{\circ}{K})\}$.

Theorem 5.4. If a sequence of random closed sets $\left\{\mathbf{S}_{n}\right\}_{n \in \mathbf{N}}$ pseudoweakly converges to a random closed set $\mathbf{S}$ and $\mathbf{T}$ and $\mathbf{T}_{n}$ are corresponding capacity functionals, then for all $K \in \mathscr{S}_{\mathbf{T}}$ we have $\lim _{n \rightarrow \infty} \mathbf{T}_{n}(K)=\mathbf{T}(K)$.

$\mathrm{P}$ r o o f. Let us prove that for all $K \in \mathscr{S}_{\mathbf{T}}$ the inequalities

$$
\begin{gathered}
\operatorname{lim\operatorname {sup}} \mathbf{T}_{n}(K) \leqslant \mathbf{T}(K) \\
\liminf _{n} \mathbf{T}_{n}(K) \geqslant \mathbf{T}(K)
\end{gathered}
$$

hold. Since every compact set is closed, we have $\mathbf{T}(K)=\mathbf{T}(\operatorname{cl} K)$, where $\operatorname{cl} K$ is a closure of $K$. Now, the inclusion $K \in \mathscr{S}_{\mathbf{T}}$, Theorem 5.2, and monotonicity of the capacity functional $\mathbf{T}_{n}$ yield $\mathbf{T}(K)=\mathbf{T}(\stackrel{\circ}{K}) \leqslant$ $\liminf \operatorname{Tin}_{n}(\stackrel{\circ}{K}) \leqslant \liminf \mathbf{T}_{n}(K)$, and inequality (5.14) holds.

Analogously, Theorem 5.1 and monotonicity of the capacity functionals $\mathbf{T}_{n}$ yield $\mathbf{T}(K)=\mathbf{T}(\operatorname{cl} K) \geqslant \lim \sup _{n} \mathbf{T}_{n}(\operatorname{cl} K) \geqslant \lim \sup _{n} \mathbf{T}_{n}(K)$, and the claim follows from (5.13) and (5.14).

Theorem 5.5. If a sequence of capacity functionals $\left\{\mathbf{T}_{n}\right\}_{n \in \mathbf{N}}$ converges to the capacity functional $\mathbf{T}$, then the corresponding sequence of random closed sets $\left\{\mathbf{S}_{n}\right\}_{n \in \mathbf{N}}$ pseudo-weakly converges to a random closed set $\mathbf{S}$.

P r o of. Let $s$ be the simple function with the range $\operatorname{Rang}(s)=$ $\left\{a_{1}, \ldots, a_{m}\right\}$, such that $a_{1}<a_{2}<\cdots<a_{m}<\infty$, and disjoint sets $A_{i}$, $i=1, \ldots, a_{m}$. Let $c_{1}^{*}=a_{1}, c_{2}^{*}=a_{2} \ominus a_{1}, \ldots, c_{m}^{*}=a_{m} \ominus a_{m-1}$. Sets $C_{i}^{*}$, $i=1, \ldots, m$, are defined in the usual way, i.e., $C_{i}^{*}=\bigcup_{j=i}^{m} A_{j}$.

The definition of the pseudo-integral, the continuity of pseudo-addition and pseudo-multiplication imply

$$
\begin{aligned}
\lim _{n \rightarrow \infty} \int^{\oplus} s \odot d \mathbf{T}_{n} & =\lim _{n \rightarrow \infty}\left(c_{1}^{*} \odot \mathbf{T}_{n}\left(C_{1}^{*}\right) \oplus \cdots \oplus c_{m}^{*} \odot \mathbf{T}_{n}\left(C_{m}^{*}\right)\right) \\
& =\lim _{n \rightarrow \infty} c_{1}^{*} \odot \mathbf{T}_{n}\left(C_{1}^{*}\right) \oplus \cdots \oplus \lim _{n \rightarrow \infty} c_{m}^{*} \odot \mathbf{T}_{n}\left(C_{m}^{*}\right) \\
& =c_{1}^{*} \odot \lim _{n \rightarrow \infty} \mathbf{T}_{n}\left(C_{1}^{*}\right) \oplus \cdots \oplus c_{m}^{*} \odot \lim _{n \rightarrow \infty} \mathbf{T}_{n}\left(C_{m}^{*}\right) \\
& =c_{1}^{*} \odot \mathbf{T}\left(C_{1}^{*}\right) \oplus \cdots \oplus c_{m}^{*} \odot \mathbf{T}\left(C_{m}^{*}\right)=\int^{\oplus} s \odot d \mathbf{T}
\end{aligned}
$$

i.e., $\lim _{n \rightarrow \infty} \int^{\oplus} s \odot d \mathbf{T}_{n}=\int^{\oplus} s \odot d \mathbf{T}$ for all simple functions $s$.

Using the definition of a pseudo-integral of a continuous bounded function $f: \mathbf{R} \rightarrow[0, F]$ the claim of the theorem holds.

The following example shows that if $f$ is not a bounded function, then the convergence of the sequence of capacity functionals do not imply the $(\oplus, \odot)$-weak convergence of the sequence of random closed sets. 
E x a m p le 5.1. Let $\left\{\mathbf{X}_{n}\right\}_{n \in \mathbf{N}}$ be a sequence of random variables with distribution functions $\mathbf{F}_{n}(x)=(x /(x+1))^{n}, x>0, n \in \mathbf{N}$. Obviously, for all $x \in \mathbf{R}$, we have $\mathbf{F}_{n}(x) \rightarrow 0$ as $n \rightarrow \infty$.

Let $\left\{\mathbf{S}_{n}\right\}_{n \in \mathbf{N}}$ be a sequence of random sets, where $\mathbf{S}_{n}=\left\{\mathbf{X}_{n}\right\}$. It is known that $\mathbf{T}_{n}(K)=\mathbf{P}\left(\mathbf{X}_{n} \in K\right)$. Also, for all $K \in \mathscr{K}$,

$$
\mathbf{P}\left(\mathbf{X}_{n} \in K\right) \leqslant \mathbf{P}\left(\mathbf{X}_{n} \leqslant \max K\right)=\mathbf{F}_{n}(\max K) \rightarrow 0, \quad n \rightarrow \infty,
$$

therefore $\mathbf{T}_{n}(K) \rightarrow 0$ as $n \rightarrow \infty$ and $\mathbf{T}=0$ identically. Now, the Choquet theorem [3] ensures us existence of a (unique) random set $\mathbf{S}$ with the probability measure $\mathbf{P}_{\mathbf{S}}$ which is determined by $\mathbf{T}$. This $\mathbf{T}$ is the Dirac probability measure at $\varnothing$.

Let us consider a pseudo characteristic function of $\mathbf{R}^{+}$. It is obvious that $\mathbf{T}_{\mathbf{S}_{n}}\left(\mathbf{R}^{+}\right)=\mathbf{P}\left(\mathbf{X}_{n} \in \mathbf{R}^{+}\right)=1$ for all $n \in \mathbf{N}$, therefore from the definition of the pseudo-integral it follows that $\int{ }^{\oplus} \chi_{\mathbf{R}^{+}} \odot d \mathbf{T}_{\mathbf{S}_{n}}=\mathbf{T}_{\mathbf{S}_{n}}\left(\mathbf{R}^{+}\right)=1$. On the other hand, $\int^{\oplus} \chi_{\mathbf{R}^{+}} \odot d \mathbf{T}_{\mathbf{S}}=0$, i.e., the weak convergence based on the pseudo-integral does not hold.

\section{Weak convergence of probability measures.}

D e f i n it i o $n$ 6.1. The sequence of probability measures $\left\{\mathbf{P}_{n}\right\}_{n \in \mathbf{N}}$ weakly converges to a probability measure $\mathbf{P}$ (notation: $\mathbf{P}_{n} \Rightarrow \mathbf{P}$ ), if and only if for all $\mathbf{P}$-continuity sets $A$ we have $\lim _{n \rightarrow \infty} \mathbf{P}_{n}(A)=\mathbf{P}(A)$.

A set $A$ is called a $\mathbf{P}$-continuity set if its boundary satisfies $\mathbf{P}(\partial A)=0$.

The following theorems provide connections between the weak convergence of the sequence of probability measures induced by the sequence of random closed sets (a sequence of probability measures defined on the space $(\mathscr{F}, \Sigma(\mathscr{F})))$ and limsup and liminf of the corresponding sequence of the pseudo-integrals.

Theorem 6.1. Let $\left\{\mathbf{P}_{n}\right\}_{n \in \mathbf{N}}$ and $\mathbf{P}$ be probability measures on $(\mathscr{F}, \Sigma(\mathscr{F}))$. If $\mathbf{P}_{n} \Rightarrow \mathbf{P}$ for all bounded continuous functions $f: \mathbf{R} \rightarrow[0, \infty]$, then

$$
\limsup _{n} \int^{\oplus} f \odot d \mathbf{T}_{n} \leqslant \int^{\oplus} f \odot d \mathbf{T} .
$$

P r o o f. For all $n \in \mathbf{N}$, we have $\mathbf{T}_{n}(C)=\mathbf{P}_{n}\left(\left\{\omega: \mathbf{S}_{n}(\omega) \cap C \neq \varnothing\right\}\right)=$ $\mathbf{P}_{n}\left(\mathscr{F}_{C}\right)$ and $\mathbf{T}(C)=\mathbf{P}\left(\mathscr{F}_{C}\right), C \in \mathscr{P}$. As in Theorem 5.5, let $s$ be a simple function with the range $\operatorname{Rang}(s)=\left\{a_{1}, \ldots, a_{m}\right\}$ and let $c_{1}^{*}=a_{1}$, $c_{2}^{*}=a_{2} \ominus a_{1}, \ldots, c_{m}^{*}=a_{m} \ominus a_{m-1}$. The definition of the pseudo-integral, the portmanteau theorem, and (2.1) imply

$$
\begin{aligned}
\underset{n}{\limsup } \int^{\oplus} s \odot d \mathbf{T}_{n}=\underset{n}{\limsup } \bigoplus_{i=1}^{m} c_{i}^{*} \odot \mathbf{T}_{n}\left(C_{i}^{*}\right) \\
\quad=\limsup \bigoplus_{i=1}^{m} c_{i}^{*} \odot \mathbf{P}_{n}\left(\mathscr{F}_{C_{i}^{*}}\right) \leqslant \bigoplus_{i=1}^{m} \limsup _{n} c_{i}^{*} \odot \mathbf{P}_{n}\left(\mathscr{F}_{C_{i}^{*}}\right)
\end{aligned}
$$




$$
\begin{aligned}
& =\bigoplus_{i=1}^{m} c_{i}^{*} \odot \limsup _{n} \mathbf{P}_{n}\left(\mathscr{F}_{C_{i}^{*}}\right) \leqslant \bigoplus_{i=1}^{m} c_{i}^{*} \odot \mathbf{P}\left(\mathscr{F}_{C_{i}^{*}}\right) \\
& =\bigoplus_{i=1}^{m} c_{i}^{*} \odot \mathbf{T}\left(C_{i}^{*}\right)=\int^{\oplus} s \odot d \mathbf{T},
\end{aligned}
$$

i.e., for all simple functions $s$ inequality (6.1) holds. By the definition of the pseudo-integral it follows that inequality (6.1) holds for all continuous bounded functions $f$.

Theorem 6.2. Let $\left\{\mathbf{P}_{n}\right\}_{n \in \mathbf{N}}$ and $\mathbf{P}$ be probability measures on $(\mathscr{F}, \Sigma(\mathscr{F}))$. If $\mathbf{P}\left(\mathscr{F}_{A}\right)=\mathbf{P}\left(\stackrel{\circ}{F}_{A}\right)$ for all sets $A \in \Sigma(\mathscr{F})$ and $\mathbf{P}_{n} \Rightarrow \mathbf{P}$, then, for all continuous bounded functions $f: \mathbf{R} \rightarrow[0, \infty]$,

$$
\liminf _{n} \int^{\oplus} f \odot d \mathbf{T}_{n} \geqslant \int^{\oplus} f \odot d \mathbf{T} .
$$

P r o o f. We have $\mathbf{T}_{n}(C)=\mathbf{P}_{n}\left(\mathscr{F}_{C}\right)$ for all $n \in \mathbf{N}$ and $\mathbf{T}(C)=$ $\mathbf{P}\left(\mathscr{F}_{C}\right), C \in \mathscr{P}$. For the simple function $s$, from the definition of the pseudo-integral, Theorem 1.1, monotonicity of capacity functional, and (2.2) it follows that

$$
\begin{aligned}
\liminf _{n} \int^{\oplus} s \odot d \mathbf{T}_{n}=\liminf _{n} \bigoplus_{i=1}^{m} c_{i}^{*} \odot \mathbf{T}_{n}\left(C_{i}^{*}\right) \\
=\liminf _{n} \bigoplus_{i=1}^{m} c_{i}^{*} \odot \mathbf{P}_{n}\left(\mathscr{F}_{C_{i}^{*}}\right) \geqslant \bigoplus_{i=1}^{m} \liminf _{n} c_{i}^{*} \odot \mathbf{P}_{n}\left(\mathscr{F}_{C_{i}^{*}}\right) \\
=\bigoplus_{i=1}^{m} c_{i}^{*} \odot \liminf _{n} \mathbf{P}_{n}\left(\mathscr{F}_{C_{i}^{*}}\right) \geqslant \bigoplus_{i=1}^{m} c_{i}^{*} \odot \liminf _{n} \mathbf{P}_{n}\left(\stackrel{\circ}{F}_{C_{i}^{*}}\right) \\
\geqslant \bigoplus_{i=1}^{m} c_{i}^{*} \odot \mathbf{P}\left(\stackrel{\circ}{\mathscr{F}}_{C_{i}^{*}}\right)=\bigoplus_{i=1}^{m} c_{i}^{*} \odot \mathbf{P}\left(\mathscr{F}_{C_{i}^{*}}\right)=\bigoplus_{i=1}^{m} c_{i}^{*} \odot \mathbf{T}\left(C_{i}^{*}\right)=\int^{\oplus} s \odot d \mathbf{T},
\end{aligned}
$$

i.e., inequality (6.2) holds for all simple functions. By the definition of the pseudo-integral, (6.2) holds for all continuous bounded functions $f$.

Corollary 6.1. Let $\left\{\mathbf{P}_{n}\right\}_{n \in \mathbf{N}}$ and $\mathbf{P}$ be probability measures on $(\mathscr{F}, \Sigma(\mathscr{F}))$. If for all sets $A \in \Sigma(\mathscr{F})$ and the probability measure $\mathbf{P}$ we have $\mathbf{P}\left(\mathscr{F}_{A}\right)=\mathbf{P}\left(\stackrel{\circ}{\mathscr{F}}_{A}\right)$, then the weak convergence $\mathbf{P}_{n} \Rightarrow \mathbf{P}$ implies that the corresponding sequence of random closed sets $\left\{\mathbf{S}_{n}\right\}_{n \in \mathbf{N}}$ pseudo-weakly converges to random closed set $\mathbf{S}$.

\section{REFERENCES}

1. Benvenuti P., Mesiar R., Vivona D. Monotone set functions-based integrals. - Handbook of Measure Theory, v. 2. Ed. by E. Pap. Amsterdam: North-Holland, 2002, p. 1329-1379.

2. Биллингсли П. Сходимость вероятностных мер. М.: Наука, 1977, 351 с.

3. Choquet G. Theory of capacities. - Ann. Inst. Fourier, 1953, v. 5, p. 131-295. 
4. Denneberg D. Non-Additive Measure and Integral. Dordrecht: Kluwer, 1994, 178 p.

5. Фукс Л. Частично упорядоченные алгебраические системы. М.: Мир, 1965, 342 с.

6. Grbić T., Pap E. Pseudo-weak convergence of the random sets defined by a pseudointegral based on non-additive measure. - International Workshop «Idempotent and Tropical Mathematics and Problems of Mathematical Physics» (Moscow, 2007), v. I. Ed. by G. L. Litvinov, V. P. Maslov, and S. N. Sergeev. Moscow: Independent University of Moscow, 2007, p. 72-77.

7. Goutsias J. Modeling random shapes: an introduction to the random closed set theory. Technical Report JHU/ECE, 1990.

8. Jupp D. L. B., Strahler A.H., Woodcock C.E. Autocorrelation and regularization in digital images. I. Basic theory. - IEEE Trans. Geoscience and Remote Sensing, 1988, v. 26, p. $463-473$.

9. Jupp D. L. B., Strahler A.H., Woodcock C.E. Autocorrelation and regularization in digital images. II. Simple image models. - IEEE Trans. Geoscience and Remote Sensing, 1989, v. 27, p. 247-258.

10. Kendall D. G. Foundations of a theory of random sets. - Stochastic Geometry. Ed. by E. F. Harding and D. G. Kendall. London: Wiley, 1974, p. 322-376.

11. Klein E., Thompson A. C. Theory of Correspondences. New York: Wiley, 1984, 256 p.

12. Klement E. P., Mesiar R., Pap E. Triangular Norms. Dordrecht: Kluwer, 2000, 385 p.

13. Kuich W., Salomaa A. Semirings, Automata, Languages. Berlin: Springer-Verlag, $1986,374 \mathrm{p}$.

14. Litvinov G. L. The Maslov dequantization, idempotent and tropical mathematics: a very brief introduction. - Idempotent Mathematics and Mathematical Physics. Providence: Amer. Math. Soc., 2005, p. 1-17. (Contemp. Math., v. 377.)

15. Litvinov G. L., Maslov V.P. (Eds.). Idempotent Mathematics and Mathematical Physics. Providence: Amer. Math. Soc., 2005. (Contemp. Math., v. 377.)

16. Maslov V.P., Samoborskiu S. N. (Eds.). Idempotent Analysis. Providence: Amer. Math. Soc., 1992. (Adv. in Soviet Math., v. 13.)

17. Матерон Ж. Случайные множества и интегральная геометрия. М.: Мир, 1978, $318 \mathrm{c}$.

18. Молчанов И. С. Об одном обобщении теоремы Шоке для случайных множеств с данным классом реализаций. - Теория вероятн. и матем. статист., 1984, т. 28, c. $99-106$.

19. Молчанов И. С. Эмпирическое оценивание квантилей распределений случайных замкнутых множеств. - Теория вероятн. и ее примен., 1990, т. 35, в. 3, с. 586-592.

20. Молчанов И. С. Состоятельная оценка параметров булевых моделей случайных замкнутых множеств. - Теория вероятн. и ее примен., 1991, т. 36, в. 3, с. 580-587.

21. Molchanov I. S. Limit Theorems for Unions of Random Closed Sets. Berlin: SpringerVerlag, 1993, 157 p. (Lecture Notes in Math., v. 1561.)

22. Molchanov I. Theory of Random Sets. London: Springer-Verlag, 2005, 488 p.

23. Nguyen H. T. Choquet weak convergence of capacity functionals of random sets. Soft Methodology and Random Information Systems. Berlin: Springer-Verlag, 2004, p. 19-31.

24. Nguyen H. T., Bouchon-Meunier B. Random sets and large deviations principle as a foundation for possibility measures. - Soft Computing, 2004, v. 8, p. 61-70.

25. Pap E. Null-Additive Set Functions. Dordrecht: Kluwer, 1995, 315 p.

26. Pap E. Pseudo-additive measures and their applications. - Handbook of Measure Theory, v. II. Ed. by E. Pap. Amsterdam: North-Holland, 2002, p. 1403-1468.

27. Pap E., Grbić T., Nedović Lj., Ralević N. M. Weak convergence of random sets. 3rd Serbian-Hungarian Joint Symposium on Intelligent Systems (Subotica, 2005), p. $73-80$.

28. Puhalskii A. Large Deviations and Idempotent Probability. Boca Raton: Chapman \& Hall/CRC, 2001, 500 p.

29. Serra J. Image Analysis and Mathematical Morphology. London: Academic Press, $1984,610 \mathrm{p}$. 